\title{
A humanized yeast-based toolkit for monitoring phosphatidylinositol 3-kinase activity at both single cell and population levels
}

\author{
Julia María Coronas-Serna ${ }^{1}$, Teresa Fernández-Acero ${ }^{1}$, María Molina ${ }^{1}$ and Víctor J. Cid ${ }^{1, *}$ \\ ${ }^{1}$ Departamento de Microbiología y Parasitología, Facultad de Farmacia. Universidad Complutense de Madrid e Instituto Ramón y \\ Cajal de Investigaciones Sanitarias (IRYCIS). \\ * Corresponding Author: \\ Víctor J. Cid, Departamento de Microbiología y Parasitología, Facultad de Farmacia, Universidad Complutense de Madrid, \\ Pza. Ramón y Cajal s/n. 28040-Madrid; Tel: +34 91 3941888; Fax: +34 91 3941745; E-mail: vicjcid@ucm.es
}

\begin{abstract}
Phosphatidylinositol 3-kinase (PI3K) is a key regulator of phosphoinositide-dependent signaling in mammalian cells and its dysfunction is related to multiple syndromes, including cancer. By heterologous expression in Saccharomyces cerevisiae, we have developed a humanized yeast system as a tool for functional studies on higher eukaryotic PI3K. Here we restrict PI3K activity in yeast to specific plasma membrane (PM) microdomains by fusing the p110 $\alpha$ PI3K catalytic subunit to either a septin or an eisosome component. We engineered a Dual Reporter for PI3K (DRAPIK), useful to monitor activity on cellular membranes in vivo at a single-cell level, by simultaneous $\mathrm{PM}$ staining of the enzyme substrate (Ptdlns4,5 $\mathrm{P}_{2}$ ) with GFP and its product (PtdIns3,4,5P $\mathrm{P}_{3}$ ) with mCherry. We also developed a sensitive FLUorescence by PI3K Inhibition (FLUPI) assay based on a GFP transcriptional reporter that is turned off by PI3K activity. This reporter system proved useful to monitor PI3K inhibition in vivo by active compounds. Such novel tools were used to study the performance of yeast PM microdomain-directed PI3K. Our results show that tethering heterologous PI3K to discrete PM domains potentiates its activity on Ptdlns4,5 $\mathrm{P}_{2}$ but different locations display distinct effects on yeast growth and endocytosis.
\end{abstract}

doi: $10.15698 /$ mic2018.12.660

Received originally: 18.05.2018;

in revised form: 23.09.2018,

Accepted 04.10.2018,

Published 12.11.2018.

Keywords: PI3K, $p 110 \alpha$, phosphoinositides, Saccharomyces cerevisiae, humanized yeast, heterologous expression, fluorescent reporter, eisosomes, septins, kinase inhibitors.
Abbreviations:
DRAPIK - Dual Reporter for the Activity of PI3K,
FLUPI - FLUorescence by PI3K Inhibition,
MCC - membrane compartment
containing Can1,
PM - plasma membrane.

\section{INTRODUCTION}

Class I phosphatidylinositol 3-kinases (PI3Ks) are responsible for conversion of Ptdlns $4,5 \mathrm{P}_{2}$ into Ptdlns $3,4,5 \mathrm{P}_{3}$ in eukaryotic cells. Inhibitors of this enzymatic activity have thoroughly been sought, as its product is a second messenger involved in proliferative, anti-apoptotic and inflammatory pathways, among others [1]. By heterologous expression in the Saccharomyces cerevisiae eukaryotic unicellular model, which naturally lacks such PI3K activity, we have previously engineered a system to in vivo titrate the activity of pathologic mutant versions of components of the human PI3K pathway, namely the $\mathrm{p} 110 \alpha$ catalytic [2-4] and p85 regulatory subunits [5] that conform the class IA PI3K, its main effector Akt $[4,6]$ and its key down-regulator, the tumor suppressor PTEN $[7,8]$. In order to meet its substrate, the PI3K p110 $\alpha$ catalytic subunit must be directed to the plasma membrane (PM). In mammalian cells, spatial regulation occurs by recruitment via the p85 regulatory subunit, which recognizes phospho-Tyr residues generated by local ligand-mediated activation of tyrosine kinasecoupled receptors $[9,10]$. The PI3K effector Akt1 is subsequently anchored to the $\mathrm{PM}$ via its Ptdlns $3,4,5 \mathrm{P}_{3}$-binding $\mathrm{PH}$ domain, where it is activated by phosphorylation at different sites by PDK1 and the TORC2 complex $[10,11]$. In our yeast engineered model, PM tethering of the $\mathrm{p} 110 \alpha$ catalytic subunit is achieved by adding $\mathrm{N}$-terminal myristoylation or C-terminal CAAX prenylation signals to this protein [4, 5]. Reconstitution of class IA PI3K activity by this means results in yeast growth inhibition as a consequence of the withdrawal of essential PM Ptdlns4, $5 \mathrm{P}_{2}$ pools [12], as well as in localization and enhanced phosphorylation of Akt1 at the PM due to the production of Ptdlns3,4,5 $\mathrm{P}_{3}$ [6]. Remarkably, expression of neither naked (non PM-directed) p110 $\alpha$ nor Akt1 alone in yeast leads to growth defect, whereas co-expression of both heterologous proteins inhibits yeast growth in an Akt catalytic activ- 
ity-dependent manner as a consequence of the interference of active Akt with yeast TORC2 signaling [13]. Thus, even in the absence of the p85 regulatory subunit or artificial targeting to the yeast PM, p110 $\alpha$ can produce enough Ptdlns3,4,5P $\mathrm{P}_{3}$ to efficiently relocate and activate Akt. However, unlike myr-p110 $\alpha$ and $p 110 \alpha-C A A X$, naked $p 110 \alpha$ is not able to eliminate enough PtdIns4, $5 \mathrm{P}_{2}$ from the yeast PM to compromise cell viability $[5,7]$.

Recent evidence suggests that the yeast $\mathrm{PM}$ is organized in microdomains and that Ptdlns4,5 $\mathrm{P}_{2}$ plays a role in configuring them $[14,15]$. In turn, microdomains such as the eisosome/MCC (Membrane Compartment containing Can1) are involved in the control of Ptdlns4, $5 \mathrm{P}_{2}$ levels [1517]. Here, we have designed chimeras to fine-tune the localization of heterologous PI3K activity in the yeast PM to two specific membrane microdomains that are stable along the budding cycle: the septin collar at the bud neck and eisosomes/MCCs. Moreover, in order to determine PI3K activity in vivo at both single-cell and whole population levels, we have developed novel fluorescent protein-based reporters that accurately permit assessment of heterologous PI3K activity in the yeast model.

\section{RESULTS AND DISCUSSION}

\section{Spatial targeting of PI3K activity to specific yeast PM} microdomains

$\mathrm{N}$-terminal myristoylation or C-terminal prenylation signals, previously used to bring $\mathrm{p} 110 \alpha$ in contact to its substrate $[2,4,5]$, should indiscriminately direct PI3K activity to any location at the yeast PM. Instead, we decided to spatially modulate this activity to discrete domains in order to study the effects of locally enhanced conversion of Ptdlns $4,5 \mathrm{P}_{2}$ into Ptdlns3,4,5 $P_{3}$. Through the budding cycle, the stretch of PM that conforms the bud neck is tightly and stably marked by a highly structured ring of septin filaments consisting of Cdc3, Cdc10, Shs1, Cdc11 and Cdc12 [18]. Septins are Ptdlns4,5 $\mathrm{P}_{2}$-binding proteins and the interaction with this phosphoinositide promotes their assembly into filaments [19]. Therefore, we decided to specifically direct PI3K activity to the septin-delimited PM area. To this end we produced a chimera consisting of the Cdc10 septin fused to the N-terminus of $\mathrm{p} 110 \alpha$. The $\mathrm{Cdc} 10-\mathrm{p} 110 \alpha$ fusion protein resulted inhibitory for yeast cell growth and its toxicity relied on $\mathrm{p} 110 \alpha$ catalytic activity, as a kinase-dead Cdc10-p110 $\alpha(\mathrm{K} 802 \mathrm{R})$ chimera was tolerated (Fig. 1A). To prove that $\mathrm{Cdc10}$ in this sort of fusion was still tethered to the septin ring we developed a triple Cdc10-GFP-p110a chimeric protein. When expressed, this new chimera also inhibited yeast growth and specifically marked the bud neck (Fig. 1B-C). Furthermore, a point mutation in Cdc10 (Gly179 to Asp, known as the cdc10-11 allele) which impairs its assembly at the ring $[20,21]$, led to loss of both localization (data not shown) and toxicity (Fig. S1A). These results implied that the toxicity caused by the septin-PI3K chimera relied on both the targeting to the septin ring, where the PI3K catalytic subunit should be close to its substrate at the $\mathrm{PM}$, and on Ptdlns $4,5 \mathrm{P}_{2}$ consuming- $\mathrm{PI} 3 \mathrm{~K}$ activity. Despite the purported importance of Ptdlns $4,5 P_{2}$ in
A

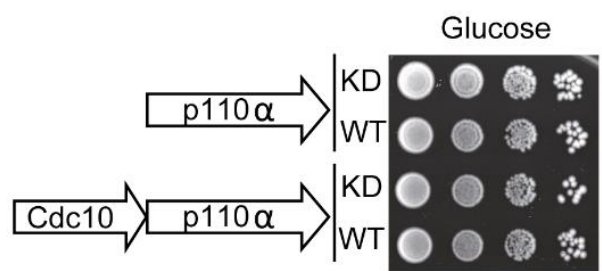

B

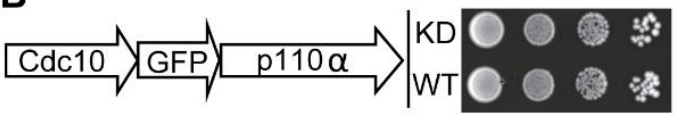

C
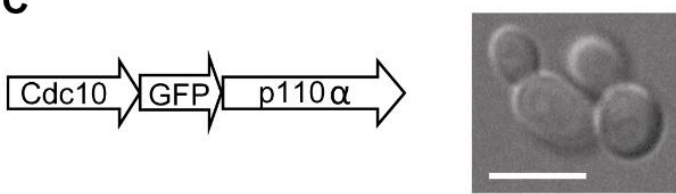

Glucose

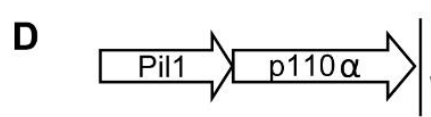

$\gamma_{W T}$

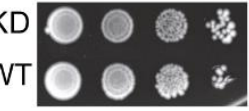

$\mathbf{E}$

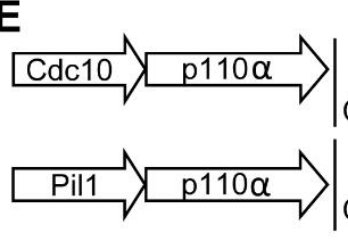

Glucose

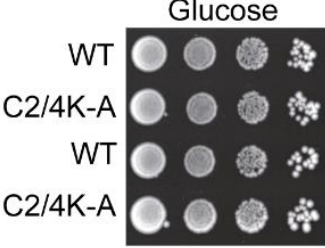

Galactose

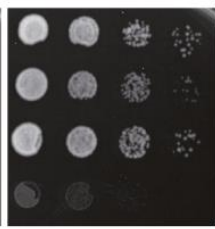

Galactose

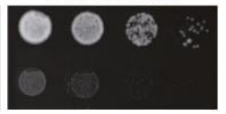

GFP

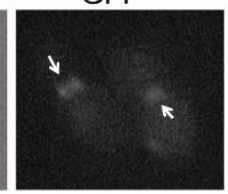

Galactose

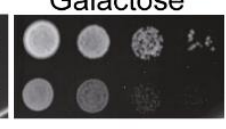

Galactose

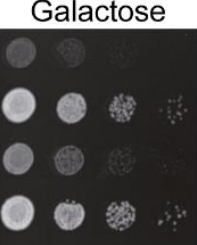

p110a

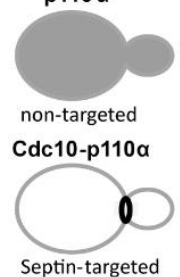

Septin-targeted

FIGURE 1: Effects on yeast growth of nontargeted and targeted $\mathrm{p} 110 \alpha$ versions. (A) Ten-fold serial dilutions of wild type YPH499 cells bearing plasmids YCpLGPI3K $\alpha(K 802 R) \quad$ [p110 $\alpha(K 802 R)], \quad Y C p L G-$

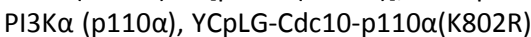
or YCpLG-Cdc10-p110 $\alpha$, respectively. Cells were cultured at $30^{\circ} \mathrm{C}$ on SD (Glucose) or SG (Galactose) agar for induction of $\mathrm{p} 110 \alpha$ expression. Expressed fusions are indicated in the corresponding sketches at the left. (B) Yeast agar drop growth assays as in (A), but of YCpLG-Cdc10-GFP-p110 $\alpha$ (K802R) or YCpLG-Cdc10-GFP-p110 $\alpha$ representative transformant clones. (C) Bright field and equivalent GFP fluorescence microscopy images of cells expressing the triple fusion protein [Cdc10-GFP-p110 $\alpha$-WT]. The arrows indicate localization at the septin ring at the mother-bud neck. The scale bar represents $5 \mu \mathrm{m}$. (D) Yeast growth assay of representative transformant clones bearing plasmids YCpLG-Pil1-p110 $\alpha$ (K802R) or YCpLG-Pil1p110 $\alpha$. (E) Growth of representative yeast clones expressing either wild type or $\mathrm{C2}$ domain mutant (C2/4K-A) p110 $\alpha$ versions of the Cdc10-p110 $\alpha$ or Pil1-p110 $\alpha$ fusion proteins, from plasmids YCpLG-Cdc10p110 $\alpha$, YCpLG-Cdc10-p110 $\quad$ C2/4K-A, YCpLG-Pil1-p110 $\alpha$ or YCpLG-Pil1-p110a $\mathrm{C} 2 / 4 \mathrm{~K}-\mathrm{A}$, as indicated. 
septin assembly [19], Cdc10-p110 $\alpha$ did not lead to phenotypes characteristic of loss of septin ring function [20], such as bud elongation (Fig. S1B). This observation hints that the Ptdlns4,5 $\mathrm{P}_{2}$ pool underlying septin filaments may not be accessible for PI3K or that $\mathrm{Cdc10}$ and PI3K are competing for the binding to this pool. We next tested whether Cdc10, when fused to the catalytically inactive $\mathrm{p} 110 \alpha$ mutant (K802R), whose expression does not affect yeast growth, was able to complement a loss-of-function cdc10-11 mutant. The Cdc10-p110 $\alpha(\mathrm{K} 802 \mathrm{R})$ chimera was not able to support growth of this mutant at the restrictive temperature (Fig. S1C), suggesting that the presence of $p 110 \alpha$ bound to its C-terminus prevented $\mathrm{Cdc} 10$ from playing its functional role at the septin filaments in spite of its proper localization.

We then studied the influence of directing PI3K activity to the MCC. With this aim, we developed a second fusion by attaching to $\mathrm{p} 110 \alpha$ the eisosome core component Pil1, an F-BAR domain-containing protein that binds curved furrow-like MCC microdomains [22]. As shown in Fig. 1D, tethering $\mathrm{p} 110 \alpha$ to the eisosomes also led to growth inhibition, although to a lesser extent than that caused by Cdc10-p110 $\alpha$. Expression of Pil1-p110 $\alpha$ did not interfere with eisosome assembly, since the localization of Sur7-GFP, a marker for these structures at the PM $[23,24]$, was apparently unaltered (Fig. S2A). Moreover, the kinase-dead Pil1-p110 $\alpha(K 802 R)$ chimera was unable to complement alterations in eisosome assembly displayed by an Isp1 $1 \Delta$ pil1 double mutant. As in the case of Cdc10$\mathrm{p} 110 \alpha(\mathrm{K} 802 \mathrm{R})$, loss of Pil1 function in Pil1-p110 $\alpha(\mathrm{K} 802 \mathrm{R})$ must be a consequence of the bulky addition of p110 to its C-terminus, as restoration of a STOP codon (by sitedirected mutagenesis in PIL1 at the site of the fusion with the $\mathrm{p} 110 \alpha$ cDNA in our expression plasmid) led to a functional Pil1 (Fig. S2B).

The $\mathrm{p} 110 \alpha \mathrm{C} 2$ domain is involved in PM recognition [25]. We previously showed that a quadruple mutation abolishing key positive charges within this domain (K410A, R412A, K413A, K416A), abrogated PI3K-dependent growth inhibition in our yeast model [5] when the interaction of $p 110 \alpha$ with the yeast PM was non-artificial, i.e., through recruitment by the regulatory subunit p $85 \alpha$. In contrast, the robust attachment of $\mathrm{p} 110 \alpha$ by the CAAX motif made this construct insensitive to these multiple $C 2$ mutations [5]. Both Cdc10 and Pil1 fusions to $\mathrm{p} 110 \alpha$ seemed to mimic $\mathrm{p} 85 \alpha$ function by bringing $\mathrm{p} 110 \alpha$ in proximity to the PM, as the $\mathrm{C} 2$ domain of $\mathrm{p} 110 \alpha$ was also necessary for the toxicity of these chimeras (Fig. 1E).

In sum, synthetically directing $\mathrm{p} 110 \alpha$ to discrete scaffolds at the yeast submembrane, such as the septin ring or the eisosomes, simulates physiological recruitment by the PI3K regulatory subunit in higher cells without apparently interfering with the assembly and function of these structures, and leads to Ptdlns4,5 $\mathrm{P}_{2}$ depletion and toxicity due to PI3K activity.
A
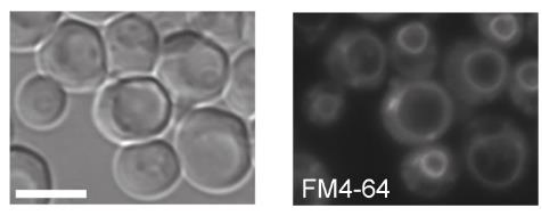

B

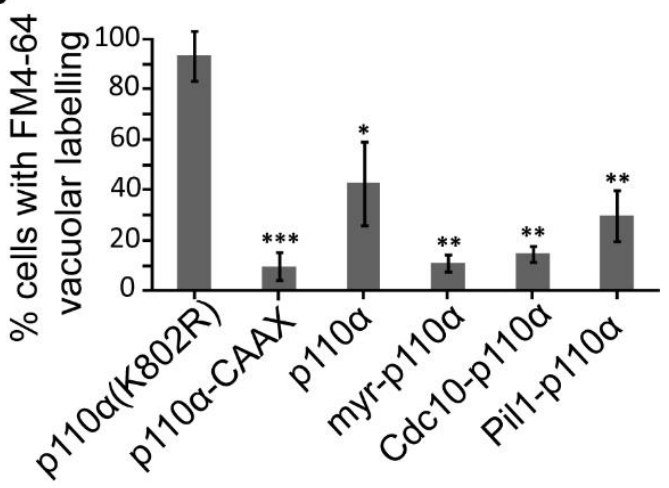

FIGURE 2: Endocytic fitness of non-targeted and various PMtargeted $\mathbf{p} \mathbf{1 1 0} \alpha$ versions. (A) Bright field (left image) or fluorescence microscopy (right image) representative images of typical FM4-64 vacuolar staining in YPH499 cells expressing the kinasedead mutant $p 110 \alpha(K 802 R), 60$ min after the addition of the vital dye. (B) Graphical representation of the percentage of cells ( $n \geq 300$ ) showing the aforementioned vacuolar staining pattern when expressing $\mathrm{p} 110 \alpha(\mathrm{K} 802 \mathrm{R}), \mathrm{p} 110 \alpha-\mathrm{CAAX}, \mathrm{p} 110 \alpha$, myr-p $110 \alpha$, Cdc10-p110 $\alpha$ and Pil1-p110 $\alpha$. Results displayed in the graphs correspond to the mean of three biological replicates performed on different clones. Error bars represent the standard deviation (SD). Asterisks $(*, * *, * * *)$ indicate a p-value $<0.05,<0.01,<$ 0.001 respectively by the Student's t-test, referred to $\mathrm{p} 110 \alpha(\mathrm{K} 802 \mathrm{R})$. Scale bars indicate $5 \mu \mathrm{m}$.

\section{Differential effects on endocytosis of PI3K activity di- rected to septins vs. eisosomes}

Due to the essential role of Ptdlns $4,5 \mathrm{P}_{2}$ in endocytic pathways [26], expression of p110 $\alpha$-CAAX in yeast leads to an impairment of endocytosis, resulting in a defective internalization of the endocytic marker FM4-64 [12]. By monitoring the incorporation of this fluorescent dye into vacuolar membranes, the final destination of the endocytic pathway, we tested whether spatial direction of PI3K activity to either the septins or eisosomes would distinctly influence endocytosis, as compared to naked (nonmembrane-directed), myristoylated (myr-p110 $\alpha$ ) or prenylated ( $110 \alpha-C A A X)$ versions of $p 110 \alpha$. All active $\mathrm{p} 110 \alpha$ versions negatively affected endocytosis, being myrp110 $\alpha$ and Cdc10-p110 $\alpha$ as efficient as p110 $\alpha$-CAAX (Fig. 2). Naked, non-PM-targeted $\mathrm{p} 110 \alpha$ significantly decreased FM4-64 vacuolar labelling but was the less efficient version of $\mathrm{p} 110 \alpha$ in doing so, as expected. Interestingly, Pil1-p110 $\alpha$ was not as competent as Cdc10-p110 $\alpha$ inhibiting endocytosis, in agreement with its lower toxicity, suggesting that tethering PI3K to the eisosomes had less impact in endocytosis than directing it to the bud neck. The role of eisosomes in endocytosis is controversial: while they were 
A

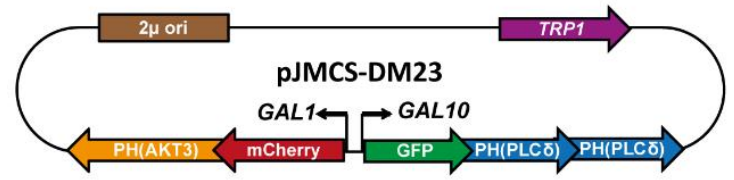

B

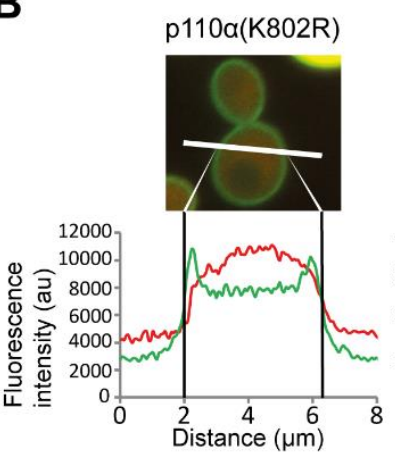

myr-p110a

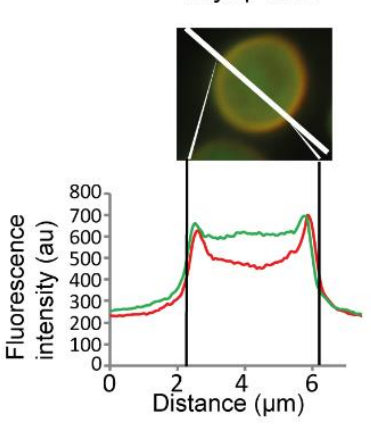

p110a-CAAX

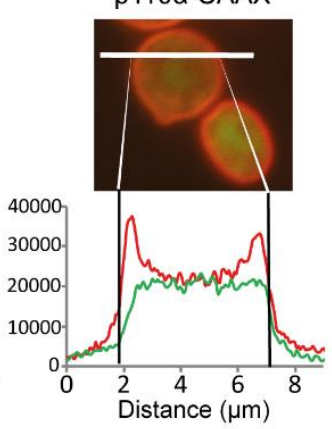

Cdc10-p110a

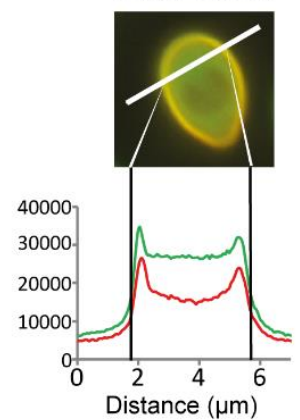

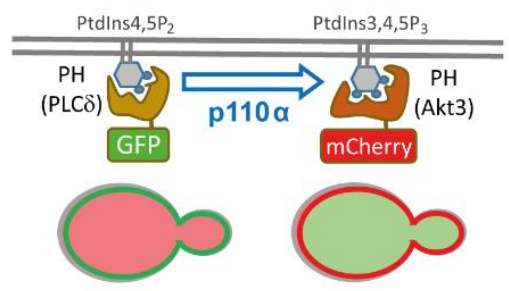

$\mathrm{p} 110 \alpha$
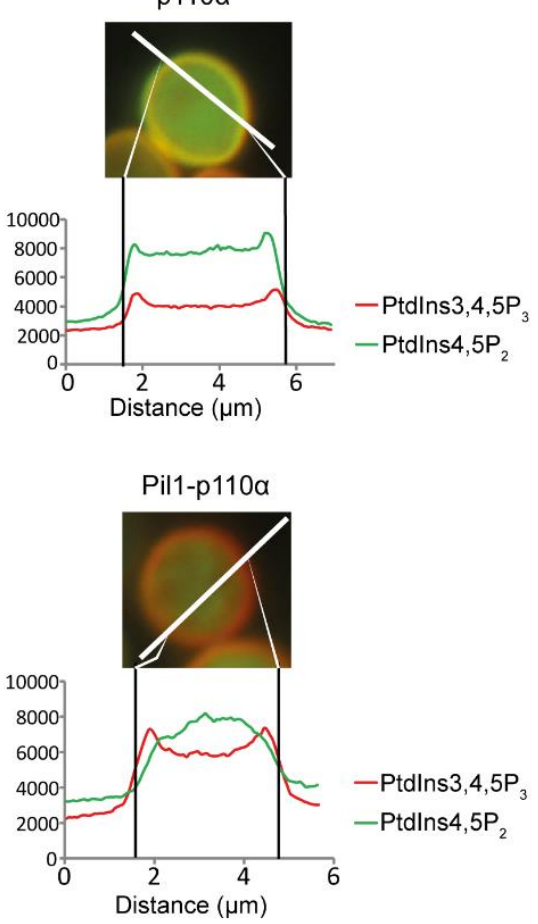

C

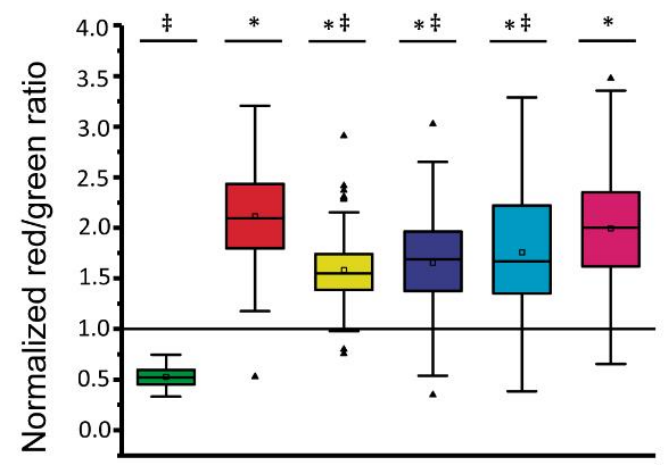

p110a(K802R)-CAAX

p110a-CAAX

p110a

myr-p110a

- Cdc10-p110a

Pil1-p110a

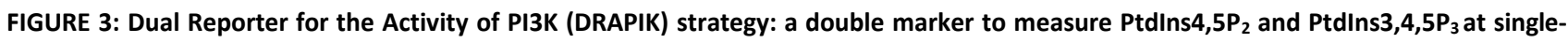
cell level. (A) Sketch of plasmid pJMCS-DM23, showing its main elements (left) and rationale and experimental design for the double marker analyses (right). Both fluorescent reporters are expressed simultaneously; yeast PM is rich in Ptdlns $4,5 \mathrm{P}_{2}$ and lacks Ptdlns $3,4,5 \mathrm{P}_{3}$, leading to recruitment of the green reporter to the PM whereas the red reporter remains cytoplasmic. Expression of PI3K should revert this situation proportionally to its enzymatic activity, the efficiency of its recruitment to the PM, and local substrate availability. (B) Representative fluorescence microscopy merged (red and green channels) images of YPH499 cells co-transformed with pJMCS-DM23 and expression plasmids for $\mathrm{p} 110 \alpha(\mathrm{K} 802 \mathrm{R}), \mathrm{p} 110 \alpha-\mathrm{CAAX}, \mathrm{p} 110 \alpha, \mathrm{myr}-\mathrm{p} 110 \alpha, \mathrm{Cdc} 10-\mathrm{p} 110 \alpha$ and Pil1-p110 $\alpha$ PI3K versions. Below, densitometric histograms showing the red (Ptdlns3,4,5 $\mathrm{P}_{3}$ ) and green (Ptdlns4,5 $\mathrm{P}_{2}$ ) fluorescence intensity in arbitrary units (au) along a transversal section of the cell, depicted above in white color, whose distance is represented in $\mu \mathrm{m}$ in abscissae. (C) Graph representing normalized red:green ratio [(Ptdlns3,4,5P $\left.{ }_{3}\right)$ vs (Ptdlns4,5P $)$ ] for a population of individually monitored randomly chosen cells ( $n=90$ per experiment) adjusted to a normal distribution. Cells co-express the same plasmids than in (B). The symbols *, $¥$ and \# express statistical significance when compared to p110 $\alpha$-KD, p110 $\alpha$ CAAX or Pil1-p110 $\alpha$, respectively (Bonferroni $p$-value $<0.01$ for two symbols and $<0.001$ for three). The symbols a and - represent the average and median values of the population; the colored boxes cluster the data between $25 \%$ and $75 \%$ of the population; the bars with a capped end (I) cluster the data between $5 \%$ and $95 \%$ of the population; the outlier data are represented by the symbol $\Delta$. 
first described as sites of endocytosis [27], several reports favor the hypothesis that they may negatively regulate endocytosis by sequestering endocytic components [28]. In fact, they have been related to down-regulation of Ptdlns4,5 $\mathrm{P}_{2}$ levels via recruitment of the phosphoinositidephosphatase Inp51/Sjl1 [17, 29]. Since Pil1-p110 $\alpha$, like Inp51/Sjl1, should locally deplete PtdIns4,5P , it is conceivable that this fusion has less impact on endocytosis as compared to other PM-targeted p110 $\alpha$ versions. In contrast, septins provide an important spatial landmark for the clustering of exo-endocytic machinery at the growing bud and at the septum [30], in consonance with a higher impact of Ptdlns4,5 $\mathrm{P}_{2}$ depletion on endocytosis from that spot.

\section{A tool for tracking PI3K activity in vivo at the single-cell level}

Once we had achieved subcellular targeting of heterologous $\mathrm{p} 110 \alpha$ to discrete PM spots, we wondered whether this could lead to loss of particular Ptdins $4,5 \mathrm{P}_{2}$ pools and spatially restricted generation of PtdIns $3,4,5 \mathrm{P}_{3}$. In order to investigate this, we developed a double fluorescent reporter vector for tracking PI3K activity in vivo at the singlecell level, by using the bidirectional expression vector pESC-TRP1 as a platform. This vector bears the GAL1GAL10 co-inducible promoters facing opposing linkers. We fused two copies of the $\mathrm{PH}(\mathrm{PLC} \delta)$ Ptdlns4,5 $\mathrm{P}_{2}$-binding domain to GFP and the $\mathrm{PH}(\mathrm{Akt} 3) \mathrm{Ptdlns3}, 4,5 \mathrm{P}_{3}$-binding do- main to mCherry (Fig. 3A). In this fashion, the yeast PM would be stained in green in the absence of PI3K activity and the red marker would be recruited to the membrane only when PI3K was locally active. We named this experimental setting Dual Reporter for the Activity of PI3K (DRAPIK).

By fluorescence microscopy, we used DRAPIK to analyze the association of the green and red signals to the PM in yeast cells upon expression of various $\mathrm{p} 110 \alpha$ versions. Representative cells are shown in Fig. 3B. As expected, cells bearing control kinase-dead $\mathrm{p} 110 \alpha(\mathrm{K} 802 \mathrm{R})$ had their PM marked only in the green channel, whereas p110 $\alpha$-CAAX fully reversed this situation and displayed a predominant peripheral red signal, suggesting that virtually all Ptdlns $4,5 \mathrm{P}_{2}$ was turned into Ptdlns $3,4,5 \mathrm{P}_{3}$, in line with the high toxicity in yeast of this version of PI3K [4]. Naked $\mathrm{p} 110 \alpha$ led to detectable conversion of Ptdlns $4,5 \mathrm{P}_{2}$ into Ptdlns $3,4,5 \mathrm{P}_{3}$, congruently with its effect on endocytosis fitness shown above in Figure 2, and with its ability to activate its downstream kinase Akt through its $\mathrm{PH}$ domain, as previously reported $[6,13]$. N-terminal myristoylation of p110 $\alpha$ led to high red signal at the PM but it did not fully eliminate PtdIns $4,5 P_{2}$, as the green signal persisted, which explains why toxicity of myr-p110 $\alpha$ in yeast is lower than that of p110 $\alpha$-CAAX [5] but higher than that of naked p110 $\alpha$. Peculiarly, direction of PI3K activity to either the septin ring or eisosomes did not lead to obvious local patterns of green to red conversion at these sites by DRAPIK
A

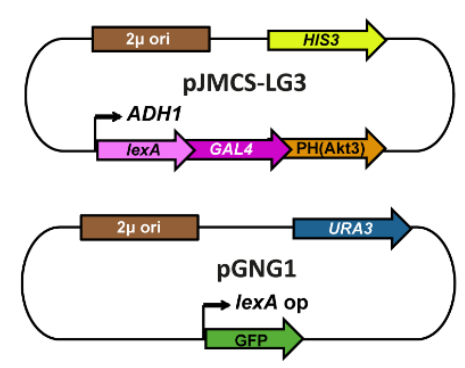

B

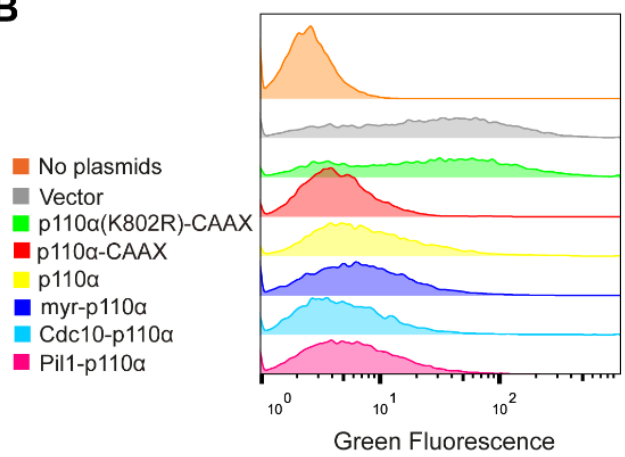

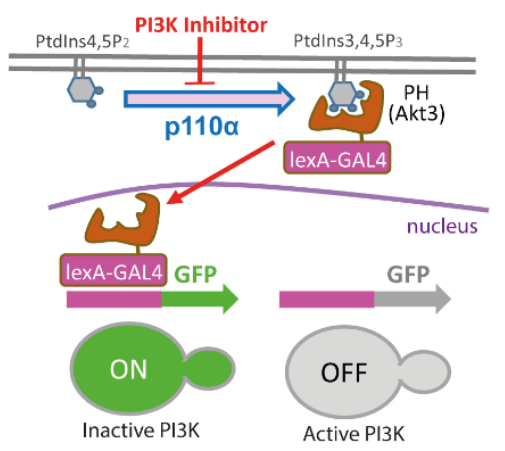

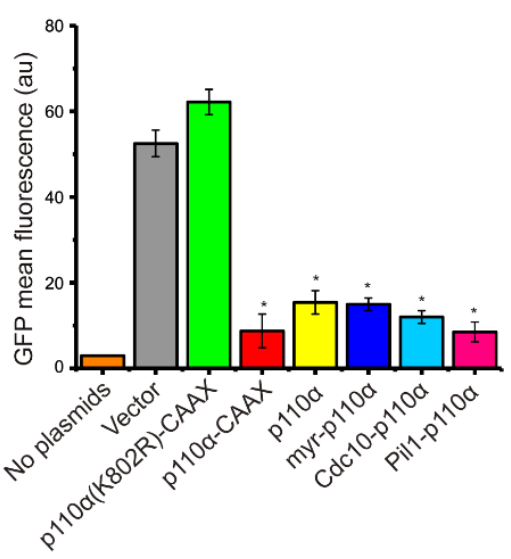

FIGURE 4: The FLUorescence by PI3K Inhibition (FLUPI) assay for the detection of heterologous PI3K activity by flow cytometry. (A) Sketches of plasmid pJMCS-LG3 bearing the transcriptional activator construct and pGNG1 (Mobitec) (left) and rationale of the system (right): the LexA-Gal4$\mathrm{PH}(\mathrm{Akt} 3)$ reporter is retained at the $\mathrm{PM}$ when the PI3K product Ptdlns $3,4,5 P_{3}$ is present. Its release due to low PI3K activity or its inhibition should proportionally lead to nuclear translocation of the chimeric transcription factor and GFP expression. (B) On the left, stacked histograms $(n=10,000)$ showing green fluorescence in abscissae of untransformed YPH499 strain (No plasmids) and triple co-transformant clones bearing pJMCS-LG3, pGNG1 and either empty YCpLG (Vector) or expressing one of the PI3K versions: p110 $\alpha($ K802R)-CAAX, p110 $\alpha$-CAAX, $\mathrm{p} 110 \alpha, \mathrm{myr}-\mathrm{p} 110 \alpha, \mathrm{Cdc} 10-\mathrm{p} 110 \alpha$ or Pil1-p110 $\alpha$. On the right, graph showing each population's mean GFP fluorescence (au) corresponding to the average of three biological replicates $(n=30,000)$. The asterisks $(*)$ indicate a Bonferroni $p$-value $<0.001$ vs. Vector; error bars represent SD. 
analysis, but rather gave also rise to general change of Ptdlns4,5 $\mathrm{P}_{2}$ into Ptdlns $3,4,5 \mathrm{P}_{3}$ along the whole PM. This likely denotes that phosphoinositides can readily diffuse in the PM even if their modifying enzymes are immobilized in particular spots.

By analyzing individually a significant number of randomly chosen cells per experiment $(n=90)$, normalizing PM vs. cytoplasmic fluorescence signals for each channel and calculating red:green ratios, we got average data for each $\mathrm{p} 110 \alpha$ version (Fig. 3C). Despite cell-to-cell variability, all $\mathrm{p} 110 \alpha$ versions except the kinase-dead control had red:green average ratios $>1$, the highest corresponding to $\mathrm{p} 110 \alpha-\mathrm{CAAX}$ and the lowest to naked $\mathrm{p} 110 \alpha$. These quantitative data are in line with our results above, indicating a correlation between PI3K-driven yeast growth inhibition and endocytosis defects, with the intriguing exception of the eisosome- and septin-directed chimeras. Pil1-p110 $\alpha$ produced a very efficient conversion of Ptdlns $4,5 \mathrm{P}_{2}$ to Ptdlns $3,4,5 P_{3}$, similar to that of $p 110 \alpha$-CAAX, whereas the septin-PI3K fusion did not eliminate all PtdIns4,5P $P_{2}$. This suggests that there is a different requirement for Ptdlns4,5 $\mathrm{P}_{2}$-dependent functions in the environment of each of the PM subdomains studied. Eisosomes are ubiquitous spots distributed around the whole PM. Thus, it is reasonable that Pil1-p110 $\alpha$ leads to a higher red signal throughout the whole $\mathrm{PM}$ since Ptdlns4,5 $\mathrm{P}_{2}-\mathrm{Ptdln} s 3,4,5 \mathrm{P}_{3}$ conversion is likely taking place all over the yeast PM. In spite of this, peculiarly, as discussed above, septin-directed PI3K activity, had a higher impact in endocytosis and viability than eisosome-directed $\mathrm{PI3K}$.

\section{A tool for the study of PI3K activity in yeast cell popula- tions that can be used to test PI3K inhibitors}

The simultaneous double phosphoinositide marker described above allows single-cell analyses but was not amenable for quantitative analyses in large cell populations. Thus, we designed a fluorescence-based strategy that would readily allow monitoring PI3K activity in yeast cultures by flow cytometry. We fused an artificial LexA-Gal4 chimeric transcriptional activator to the Ptdlns $3,4,5 \mathrm{P}_{3}$ binding PH domain of Akt3. In a strain co-transformed with a second plasmid bearing a transcriptional LexA-dependent GFP reporter and a third plasmid expressing $p 110 \alpha$, the LexA-Gal4-PH(Akt3) transcriptional activator would be sequestered at the PM by PI3K-generated PtdIns3,4,5 $\mathrm{P}_{3}$ pool, precluding GFP expression. In contrast, yeast cells lacking PI3K activity would not retain the LexA-Gal4-PH(Akt3) fusion protein at the $\mathrm{PM}$, allowing nuclear translocation and GFP expression (Fig. 4A). Since the system should lead to a gain of fluorescence upon PI3K inhibition, we named this experimental setting FLUorescence by PI3K Inhibition (FLUPI) assay. To test the system, we used as a proof-ofprinciple our collection of $\mathrm{p} 110 \alpha$ versions. All active PI3K forms, regardless of the presence or absence of PMdirecting signals, turned off GFP expression, showing a high sensitivity for detecting the presence of Ptdlns3,4,5P 3 at the PM and, therefore, PI3K activity (Fig. 4B). This assay confirmed that Pil1-p110 $\alpha$ was as efficient as p110 $\alpha$-CAAX in producing Ptdlns $3,4,5 \mathrm{P}_{3}$ in the yeast cells, in spite of its lower toxicity.

Bioassays for PI3K inhibition are useful in drug discovery. We previously reported a growth-based assay using as a platform our humanized yeast system [3]. However, fluo-
A

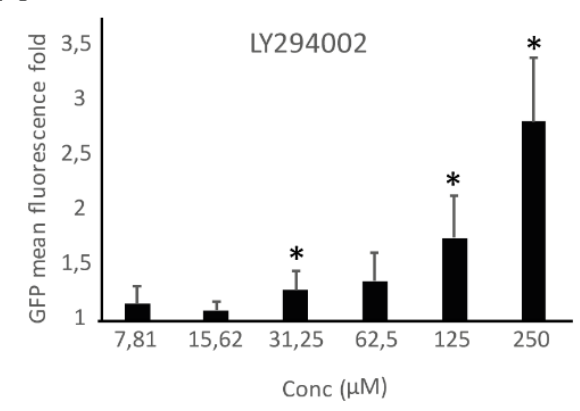

C

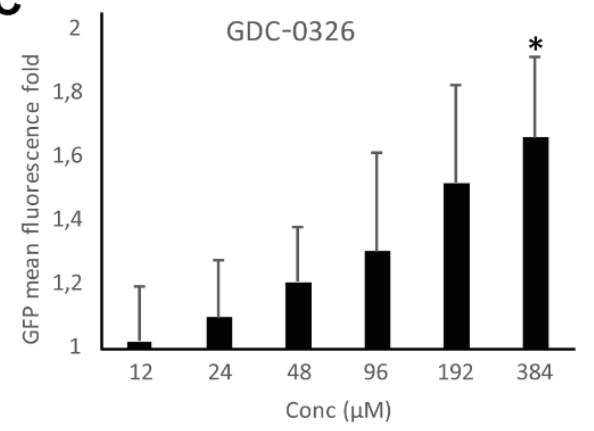

B

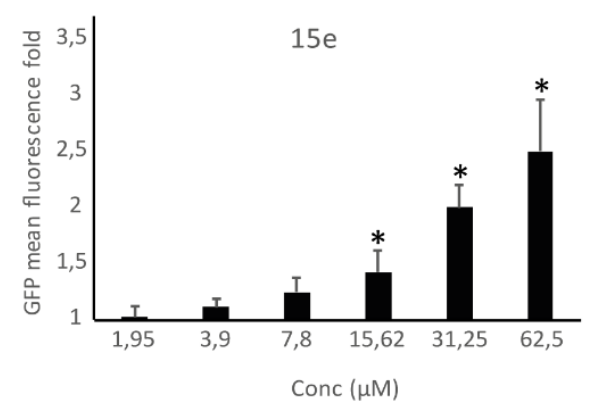

D

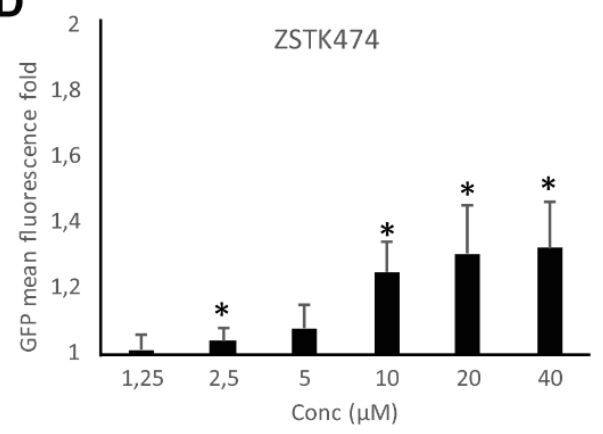

FIGURE 5: Dose-response graphs showing enhancement of fluorescence by the FLUPI assay. Triple pJMCS-LG3, pGNG1 and YCpLG-p110 $\alpha$ YPH499 transformants were analyzed by flow cytometry as in Fig. 4B in the presence of different doses of inhibitors as noted. The abscissae represent fold increase in green fluorescence of treated samples with the indicated concentrations of compounds LY294002 (A), 15e (B), GDC-0326 (C) and ZSTK474 (D) relative to their correspondent DMSO alone control (normalized to 1). Three different clones were analyzed per experiment ( $n=10,000$ cells per clone). Data correspond to the average, and error bars represent SD. Asterisks (*) mark data points that are statistically significant respective to the data of the lowest concentration of compound tested in each series, according to Student's t-test $(p<0.05)$. 
rescence-based assays are more sensitive and versatile. The above-described DRAPIK system could be useful to trace $\mathrm{PI} 3 \mathrm{~K}$ inhibition at a single-cell level but, as it requires microscopy, it would not be easily scalable to high throughput screening unless high content analysis platforms were used. On the contrary, the FLUPI assay described here might be optimum for this purpose. Since unmodified $\mathrm{p} 110 \alpha$ led to significant GFP fluorescence shutting off, we considered that this version of $p 110 \alpha$ should be more suitable for a simple PI3K inhibition assay as compared to the artificially PM-targeted $\mathrm{p} 110 \alpha$ versions tested above. Thus, we tested in a FLUPI assay based on bare p110 $\alpha$ a series of available chemical inhibitors for their ability to recover GFP fluorescence, namely LY294002 [31], the isoform-specific p110 $\alpha$ inhibitors 15e [32] and GDC0326, a benzoxazepin derivative [33], and the isoformnonspecific triazine derivative ZSTK474 [34]. For

all four compounds, a dose-dependent enhancement of fluorescence was observed in the range of concentrations tested (Fig. 5). As compared to the previously reported growth recovery assay on yeast expressing p110 $\alpha$-CAAX [3], in which we had also tested the LY294002, 15e and ZSTK474 inhibitors, the FLUPI assay also proved sensitive in the $\mu \mathrm{M}$ range for these compounds. Like in the previous setting, $15 \mathrm{e}$ was the most potent, leading to significant fluorescence increase at lower concentrations, whereas LY294002 required higher concentrations to reach the same effect. Fluorescence increase for GDC-0326 and ZSTK474 was more modest, but the latter was more efficient at low concentrations, similar to its performance in the growth recovery assay [3]. These data demonstrate the efficiency of the FLUPI bioassay to potentially screen for PI3K inhibitors on unmodified $\mathrm{p} 110 \alpha$ using fluorescencebased strategies, suitable for microfluidics or other highthroughput platforms.

In sum, we have designed a toolkit for studying class IA PI3K in a versatile humanized yeast model that permits (i) directing PI3K activity to discrete localizations at the PM, (ii) monitoring its activity at the single-cell and population levels, and (iii) studying its pharmacological inhibition in vivo. These experimental settings broaden the experimental possibilities offered by the yeast cellular model on molecular and pharmacological studies based on this key oncoprotein.

\section{MATERIALS AND METHODS}

\section{Strains, culture media and growth conditions}

Yeast strains used in this work are listed in Table S1. YPD [1\% $(\mathrm{w} / \mathrm{v})$ yeast extract, $2 \%(\mathrm{w} / \mathrm{v})$ peptone and $2 \%(\mathrm{w} / \mathrm{v})$ glucose] broth or agar was used for yeast growth as a general nonselective medium. Synthetic dextrose (SD) medium consisted of $0.17 \%$ yeast nitrogen base without amino acids, $0.5 \%$ ammonium sulfate, $2 \%$ glucose and $0.12 \%$ synthetic complete mixture drop out mix lacking the appropriate amino acids and nucleic acid bases to maintain plasmid selection. In SG (synthetic galactose) and SR (synthetic raffinose) media, glucose was respectively replaced with $2 \%(\mathrm{w} / \mathrm{v})$ galactose or $1.5 \%$ $(w / v)$ raffinose. For GAL1 promoter induction, cells were cultured in SR broth for $18 \mathrm{~h}$ at $30^{\circ} \mathrm{C}$, then the appropriate amount of these cultures was suspended into fresh SG to reach an $\mathrm{OD}_{600}$ of 0.3 , and they were incubated for additional 4 - 6 h. Spot growth assays on agar were performed as described [4].

\section{Plasmids}

Transformation of $E$. coli and yeast and other basic molecular biology methods were carried out using standard methods. All plasmids and oligonucleotides used are listed in Tables S2 and S3, respectively. CDC10 and CDC10-GFP genes were amplified by regular PCR from pLA10 plasmid [20] using Cdc10-FW, Cdc10-Rv and Cdc10-GFP-Rv primers. PIL1 was amplified from S. cerevisiae genomic DNA (BY4741) with Pil1-Fw and Pil1-Rv primers. A Gly-Ala $(5 \times)$ linker was added to the reverse primers to facilitate protein folding. All primers were flanked by $\mathrm{BamHI}$ sites and the resulting inserts were then digested with this enzyme and subsequently cloned in frame in plasmid YCpLG-PI3K $\alpha$ yielding YCpLG-Cdc10-p110 $\alpha$, YCpLG-Cdc10-GFPp110 $\alpha$ and YCpLG-Pil1-p110 $\alpha$.

Dpnl-based site directed mutagenesis was performed using the QuikChange kit (Agilent). To generate cdc10-11 allelecontaining $\mathrm{p} 110 \alpha$ fusions, the $c d c 10(G 179 D)$ mutation was introduced with primers Cdc10-G179D-Fw and Cdc10-G179DRv. The YCpLG-Pil1-STOP plasmid was obtained by introducing a stop codon in the BamHI restriction site just before the PIK3CA gene coding sequence in YCpLG-Pil1-p110 $\alpha$, using the primers STOP-p110 $\alpha-F w$ and STOP-p110 $\alpha$-Rv. p110 $\alpha$ (K802R) kinase-dead mutations and the p110 $\alpha$ C2-domain mutant C2/4K-A (K410A, R412A, K413A, K416A) were obtained with the primers previously described [5].

DRAPIK plasmid: Double marker plasmid pJMCS-DM23 was constructed by molecular cloning in the yeast expression plasmid pESC-TRP (Agilent). First, GFPx2PH(PLC $\delta$ ) was amplified by PCR with primers GFP-Fw and PH(PLC $)$-Rv using plasmid pRS426-GFP2XPH(PLCD) [35] as template. Then, it was cloned in PESC-TRP by using Spel-Bg/ll restriction sites, yielding the plasmid pJMCS-DM20. The PH domain of Akt3 (residues 1164) was amplified by PCR from pYES2-mCherry-Akt3 [13] with primers Cherry-Fw and $\mathrm{PH}(\mathrm{Akt} 3)-\mathrm{Rv}$ and finally cloned into BamHI-Nhel sites of pJMCS-DM20 to generate pJMCS-DM23.

FLUPI plasmid: To construct the pJMCS-LG3 plasmid expressing a LexA-Gal4-PH(Akt3) fusion, a Notl restriction site was artificially inserted through site-directed mutagenesis with Insert-Notl-Fw and Insert-Notl-Rv primers into the pEG202-GAL4 plasmid (MoBiTec, Germany) disrupting the stop codon of GAL4. Then the $\mathrm{PH}(\mathrm{Akt} 3)$ domain was amplified from pJMCS-DM23 using Notl-PH(Akt3)-Fw and Notl-PH(Akt3)RV primers and cloned into Notl sites of modified pEG202GAL4, to generate PJMCS-LG3.

\section{Microscopy techniques, image processing and statistical analysis}

For in vivo fluorescence microscopy (GFP or mCherry), cultures subjected to GAL1 induction were concentrated by centrifugation at $3000 \mathrm{rpm}$ for $2 \mathrm{~min}$ and visualized in an Eclipse TE2000U Nikon microscope. Digital images were captured with an Orca C4742-95-12ER camera and processed with $\mathrm{HCl}$ mage software (Hamamatsu, Hamamatsu, Japan). Endocytosis dynamics was monitored by staining with FM4-64 (Fischer Scientific) as previously reported [36].

For DRAPIK assays, fluorescence microscopy images of cells co-transformed with pJMCS-DM23 and various YCpLG-p110 $\alpha$ 
versions were analyzed as follows: measurements of both red (Ptdlns3,4,5 $\mathrm{P}_{3}$ ) and green (Ptdlns4,5 $\mathrm{P}_{2}$ ) fluorescence intensity through a line across one single cell were taken with FiJi software [37]. These values were displayed as single-cell densitometric histograms. As a quantitative approach, the "normalized red:green ratio" was calculated by measuring the fluorescence signal for both red and green channels with $\mathrm{HClmage}$ in two points at opposite sides of the PM and in one in cytoplasm. Then, for each channel, each PM fluorescence signal was normalized against the corresponding cytoplasmic fluorescence signal from the same cell to generate two PM:cytoplasm ratios per cell. The average between them becomes the final "normalized red:green ratio" for each cell. Thirty randomly chosen cells were analyzed for each culture and triplicates of three different clones were analyzed. Thus, a total of 90 cells were analyzed per $p 110 \alpha$ version assayed. A two-way ANOVA using Bonferroni test was performed with Origin Pro statistics software (OriginLab Corp., Northampton, MA, USA).

\section{Flow cytometry}

For FLUPI experiments, YPH499 yeast cells bearing pJMCS-LG3, pGNG1 and various YCpLG-p110 $\alpha$ versions were cultured as for fluorescence microscopy analysis. For GFP expression analyses, cells were collected after 5 hours of galactose induction, fixed with $4 \%$ formaldehyde in PBS, washed with PBS and then analyzed by flow cytometry in Guava easyCyte or CELLQuest 3.3 (Becton Dickinson) flow cytometers, acquiring green fluorescence through a $488 \mathrm{~nm}$ excitation laser and a 525/30 BP emission filter (BFP). Data were processed using FlowJo software (FlowJo LLC, Ashland, OR, USA). In order to calculate statistical significance, a two-way ANOVA using Bonferroni test was performed with Origin Pro statistics software (OriginLab, Northampton, MA, USA).

For p110 $\alpha$ inhibition assays, two-fold serial dilutions of $15 \mathrm{e}$ ("PI3K $\alpha$ inhibitor II", Echelon Biosciences, Salt Lake City, UT, USA), LY294002 (Echelon), GDC-0326 (Selleckchem, Houston, TX, USA) and ZSTK474 (Selleckchem), were performed in SG media. All the compounds were previously dissolved in dimethylsulfoxide (DMSO, Sigma-Aldrich) at stock concentrations of $32 \mathrm{mM}, 29 \mathrm{mM}, 100 \mathrm{mM}$ and $50 \mathrm{mM}$, respectively. A negative control containing the maximum concentration of DMSO used in each assay was included. $200 \mu \mathrm{L}$ of the yeast preinocula adjusted to an $\mathrm{OD}_{600}$ of 1 were added to every tube before incubation in a shaker at $30^{\circ} \mathrm{C}$ for $5 \mathrm{~h}$. Inhibition assays were carried out as biological triplicates. Cells were collected and processed as mentioned above. At least 10,000 cells were

\section{REFERENCES}

1. Fruman DA, Chiu H, Hopkins BD, Bagrodia S, Cantley LC, and Abraham RT (2017). The PI3K pathway in human disease. Cell 170(4): 605635. doi: 10.1016/j.cell.2017.07.029

2. Andrés-Pons A, Rodríguez-Escudero I, Gil A, Blanco A, Vega A, Molina $M$, Pulido R, and Cid VJ (2007). In vivo functional analysis of the counterbalance of hyperactive phosphatidylinositol 3-kinase p110 catalytic oncoproteins by the tumor suppressor PTEN. Cancer Res 67(20): 9731-9739. doi: 10.1158/0008-5472.can-07-1278

3. Fernández-Acero T, Rodráguez-Escudero I, Vicente F, Monteiro MC, Tormo JR, Cantizani J, Molina M, and Cid VJ (2012). A yeast-based in vivo bioassay to screen for class I phosphatidylinositol 3-kinase specif- analysed for each experiment. A green fluorescence ratio was obtained by dividing each raw value of mean GFP fluorescence at every concentration by the raw value of the DMSO control for each clone. Statistical significance was calculated by Student's t-test.

\section{ACKNOWLEDGEMENTS}

We are grateful to J. Berenguer, M. Mencía and A. Hidalgo (Universidad Autónoma de Madrid, Spain) for sharing reagents and useful discussion. We also wish to acknowledge C. Stefan for sharing the PH(PLCD)-GFP reporter, I. Rodríguez-Escudero, H. Martín and other U3/SignalYeast lab members for materials and discussion, E. Román for help with flow cytometry, and the Genomics and Flow Cytometry services at Universidad Complutense de Madrid (UCM) for sequencing and flow cytometry analyses, respectively. This work was supported by grants $\mathrm{BIO2013-}$ 44112-P and BIO2016-75030-P from Ministerio de Economía y Competitividad (Spain) and B2017/BMD-3691 (InGEMICS-CM) from Comunidad Autónoma de Madrid (CM; Spain). J.M.C-S is supported by a predoctoral contract from UCM and T.F-A is granted by BIO2013-44112-P and BIO2016-75030-P.

\section{SUPPLEMENTAL MATERIAL}

All supplemental data for this article are available online at www.microbialcell.com.

\section{CONFLICT OF INTEREST}

The authors declare no conflict of interest.

\section{COPYRIGHT}

(C) 2018 Coronas-Serna et al. This is an open-access article released under the terms of the Creative Commons Attribution (CC BY) license, which allows the unrestricted use, distribution, and reproduction in any medium, provided the original author and source are acknowledged.

Please cite this article as: Julia María Coronas-Serna, Teresa Fernández-Acero, María Molina and Víctor J. Cid (2018). A humanized yeast-based toolkit for monitoring phosphatidylinositol 3-kinase activity at both single cell and population levels. Microbial Cell 5(12): 545-554. doi: 10.15698/mic2018.12.660

ic inhibitors. J Biomol Screen 17(8): 1018-1029. doi: 10.1177/1087057112450051

4. Rodríguez-Escudero I, Roelants FM, Thorner J, Nombela C, Molina $M$, and Cid VJ (2005). Reconstitution of the mammalian PI3K/PTEN/Akt pathway in yeast. Biochem J 390(Pt 2): 613-623. doi: 10.1042/bj20050574

5. Oliver MD, Fernández-Acero T, Luna S, Rodríguez-Escudero I, Molina M, Pulido R, and Cid VJ (2017). Insights into the pathological mechanisms of p85alpha mutations using a yeast-based phosphatidylinositol 3-kinase model. Biosci Rep 37(2). doi: 10.1042/bsr20160258 
6. Rodríguez-Escudero I, Andrés-Pons A, Pulido R, Molina M, and Cid VJ (2009). Phosphatidylinositol 3-kinase-dependent activation of mammalian protein kinase B/Akt in Saccharomyces cerevisiae, an in vivo model for the functional study of Akt mutations. J Biol Chem 284(20): 13373-13383. doi: 10.1074/jbc.M807867200

7. Rodríguez-Escudero I, Fernández-Acero T, Bravo I, Leslie NR, Pulido R, Molina M, and Cid VJ (2015). Yeast-based methods to assess PTEN phosphoinositide phosphatase activity in vivo. Methods 77-78(172179. doi: 10.1016/j.ymeth.2014.10.020

8. Rodríguez-Escudero I, Oliver MD, Andrés-Pons A, Molina M, Cid VJ, and Pulido $R$ (2011). A comprehensive functional analysis of PTEN mutations: implications in tumor- and autism-related syndromes. Hum Mol Genet 20(21): 4132-4142. doi: 10.1093/hmg/ddr337

9. Liu S, Knapp S, and Ahmed AA (2014). The structural basis of PI3K cancer mutations: from mechanism to therapy. Cancer Res 74(3): 641646. doi: 10.1158/0008-5472.CAN-13-2319

10. Mayer IA, and Arteaga CL (2016). The PI3K/AKT pathway as a target for cancer treatment. Annu Rev Med 67: 11-28. doi: 10.1146/annurev-med-062913-051343

11. Roelants FM, Leskoske KL, Martinez Marshall MN, Locke MN, and Thorner J (2017). The TORC2-dependent signaling network in the yeast Saccharomyces cerevisiae. Biomolecules 7(3). doi: 10.3390/biom7030066

12. Fernández-Acero $\mathrm{T}$, Rodríguez-Escudero $\mathrm{I}$, Molina $\mathrm{M}$, and Cid VJ (2015). The yeast cell wall integrity pathway signals from recycling endosomes upon elimination of phosphatidylinositol $(4,5)$ bisphosphate by mammalian phosphatidylinositol 3-kinase. Cell Signal 27(11): 2272-2284. doi: 10.1016/j.cellsig.2015.08.004

13. Rodríguez-Escudero I, Fernández-Acero T, and Cid VJ, and Molina $M$ (2018). Heterologous mammalian Akt disrupts plasma membrane homeostasis by taking over TORC2 signaling in Saccharomyces cerevisiae. Sci Rep 8(1): 7732. doi: 10.1038/s41598-018-25717-w

14. Malinsky J, Opekarová M, and Tanner W (2010). The lateral compartmentation of the yeast plasma membrane. Yeast 27(8): 473-478. doi 10.1002/yea.1772

15. Bartlett K, Gadila SK, Tenay B, McDermott H, Alcox B, and Kim K (2015). TORC2 and eisosomes are spatially interdependent, requiring optimal level of phosphatidylinositol 4, 5-bisphosphate for their integrity. J Biosci 40(2): 299-311. PMID: 25963258

16. Berchtold D, Piccolis M, Chiaruttini N, Riezman I, Riezman H, Roux A, Walther TC, and Loewith R (2012). Plasma membrane stress induces relocalization of SIm proteins and activation of TORC2 to promote sphingolipid synthesis. Nat Cell Biol 14(5): 542-547. doi: $10.1038 /$ ncb2480

17. Fröhlich F, Christiano R, Olson DK, Alcázar-Román A, DeCamilli $P$, and Walther TC (2014). A role for eisosomes in maintenance of plasma membrane phosphoinositide levels. Mol Biol Cell 25(18): 2797-2806. doi: 10.1091/mbc.E13-11-0639

18. Glomb O, and Gronemeyer T (2016). Septin organization and functions in budding yeast. Frontiers Cell Dev Biol 4:123. doi: 10.3389/fcell.2016.00123

19. Bertin A, McMurray MA, Thai L, Garcia G, 3rd, Votin V, Grob P, Allyn T, Thorner J, and Nogales E (2010). Phosphatidylinositol-4,5bisphosphate promotes budding yeast septin filament assembly and organization. J Mol Biol 404(4): 711-731. doi: 10.1016/j.jmb.2010.10.002

20. Cid VJ, Adamiková L, Cenamor R, Molina M, Sánchez M, and Nombela $C$ (1998). Cell integrity and morphogenesis in a budding yeast septin mutant. Microbiology 144(12): 3463-3474. doi: 10.1099/00221287-144-12-3463
21. Weems AD, Johnson CR, Argüeso JL, and McMurray MA (2014). Higher-order septin assembly is driven by GTP-promoted conformational changes: evidence from unbiased mutational analysis in Saccharomyces cerevisiae. Genetics 196(3): 711-727. doi: 10.1534/genetics.114.161182

22. Olivera-Couto A, Grana M, Harispe L, and Aguilar PS (2011). The eisosome core is composed of BAR domain proteins. Mol Biol Cell 22(13): 2360-2372. doi: 10.1091/mbc.E10-12-1021

23. Grossmann G, Opekarová M, Malinsky J, Weig-Meckl I, and Tanner W (2007). Membrane potential governs lateral segregation of plasma membrane proteins and lipids in yeast. EMBO J 26(1): 1-8. doi: 10.1038/sj.emboj.7601466

24. Douglas LM, and Konopka JB (2014). Fungal membrane organization: the eisosome concept. Annu Rev Microbiol 68: 377-393. doi: 10.1146/annurev-micro-091313-103507

25. Huang CH, Mandelker D, Gabelli SB, and Amzel LM (2008). Insights into the oncogenic effects of PIK3CA mutations from the structure of p110alpha/p85alpha. Cell Cycle 7(9): 1151-1156. doi: 10.4161/cc.7.9.5817

26. De Craene JO, Bertazzi DL, Bar S, and Friant S (2017). Phosphoinositides, major actors in membrane trafficking and lipid signaling pathways. Int J Mol Sci 18(3). doi: 10.3390/ijms18030634

27. Walther TC, Brickner JH, Aguilar PS, Bernales S, Pantoja C, and Walter $P$ (2006). Eisosomes mark static sites of endocytosis. Nature 439(7079): 998-1003. doi: 10.1038/nature04472

28. Grossmann G, Malinsky J, Stahlschmidt W, Loibl M, Weig-Meckl I, Frommer WB, Opekarová $M$, and Tanner W (2008). Plasma membrane microdomains regulate turnover of transport proteins in yeast. J Cell Biol 183(6): 1075-1088. doi: 10.1083/jcb.200806035

29. Kabeche R, Roguev A, Krogan NJ, and Moseley JB (2014). A Pil1Sle1-Syj1-Tax4 functional pathway links eisosomes with $\mathrm{PI}(4,5) \mathrm{P} 2$ regulation. J Cell Sci 127(6): 1318-1326. doi: 10.1242/jcs.143545

30. Okada S, Leda M, Hanna J, Savage NS, Bi E, and Goryachev AB (2013). Daughter cell identity emerges from the interplay of Cdc42, septins, and exocytosis. Dev Cell 26(2): 148-161. doi: 10.1016/j.devcel.2013.06.015

31. Vlahos CJ, Matter WF, Hui KY, and Brown RF (1994). A specific inhibitor of phosphatidylinositol 3-kinase, 2-(4-morpholinyl)-8-phenyl4H-1-benzopyran-4-one (LY294002). J Biol Chem 269(7): 5241-5248. PMID: 8106507

32. Hayakawa M, Kaizawa $H$, Moritomo H, Koizumi T, Ohishi T, Okada M, Ohta M, Tsukamoto S, Parker P, Workman P, and Waterfield M (2006). Synthesis and biological evaluation of 4-morpholino-2phenylquinazolines and related derivatives as novel PI3 kinase p110alpha inhibitors. Bioorg Med Chem 14(20): 6847-6858. doi: 10.1016/j.bmc.2006.06.046

33. Heffron TP, Heald RA, Ndubaku C, Wei B, Augistin M, Do S, Edgar K, Eigenbrot C, Friedman L, Gancia E, Jackson PS, Jones G, Kolesnikov A, Lee LB, Lesnick JD, Lewis C, McLean N, Mortl M, Nonomiya J, Pang J, Price S, Prior WW, Salphati L, Sideris S, Staben ST, Steinbacher S, Tsui V, Wallin J, Sampath D, and Olivero AG (2016). The Rational Design of Selective Benzoxazepin Inhibitors of the alpha-Isoform of Phosphoinositide 3-Kinase Culminating in the Identification of (S)-2-((2-)(1Isopropyl-1H-1,2,4-triazol-5-yl)-5,6-dihydrobenzo[f]imidazo[1,2-d][1 ,4]oxazepin-9-yl)oxy)propanamide (GDC-0326). J Med Chem 59(3): 985-1002. doi: 10.1021/acs.jmedchem.5b01483

34. Yaguchi S, Fukui Y, Koshimizu I, Yoshimi H, Matsuno T, Gouda H, Hirono S, Yamazaki K, and Yamori T (2006). Antitumor activity of ZSTK474, a new phosphatidylinositol 3-kinase inhibitor. J Natl Cancer Inst 98(8): 545-556. doi: 10.1093/jnci/djj133 
35. Stefan CJ, Audhya A, and Emr SD (2002). The yeast synaptojaninlike proteins control the cellular distribution of phosphatidylinositol (4,5)-bisphosphate. Mol Biol Cell 13(2): 542-557. doi: 10.1091/mbc.01-10-0476

36. Vida TA, and Emr SD (1995). A new vital stain for visualizing vacuolar membrane dynamics and endocytosis in yeast. J Cell Biol 128(5): 779-792. PMID: 7533169
37. Schindelin J, Arganda-Carreras I, Frise E, Kaynig V, Longair M, Pietzsch T, Preibisch S, Rueden C, Saalfeld S, Schmid B, Tinevez JY, White DJ, Hartenstein V, Eliceiri K, Tomancak P, and Cardona A (2012). Fiji: an open-source platform for biological-image analysis. Nat Methods 9(7): 676-682. doi: 10.1038/nmeth.2019 